A Self-Assembled Multivalent Pseudopolyrotaxanes

$$
\begin{gathered}
\text { for } \\
\text { Binding Galectin-1 }
\end{gathered}
$$

Alshakim Nelson, Jason M. Belitsky, Sébastien Vidal, C. Steven Joiner,

Linda G. Baum,* and J. Fraser Stoddart*

\title{
Journal of the American Chemical Society
}

Supporting Information

(28 pages) 
We summarize here the preparation of the trivalent glycoclusters $\mathbf{8}, \mathbf{9}$, and $\mathbf{1 0}$, the chitosan polymer $\mathbf{1 1}$, the polyviologen polymer $\mathbf{2}$, the pseudopolyrotaxane $\mathbf{3}$, and the agglutination assay used to evaluate the lactoside-bearing compounds.

Synthesis of the Trifunctional Core Compounds. The trifunctional core compound $\mathbf{4}$ was prepared as described in the literature. ${ }^{52}$ The phloroglucinol-based core was prepared (Scheme 3 ) in three steps from phloroglucinol. The reaction of phloroglucinol with ethylene carbonate gave the tris-alcohol 12, and subsequent tosylation afforded the tris-tosylate $13 .{ }^{53}$ Displacement of the tosylates using $N$-benzyl- $N$-methylamine, followed by hydrogenation of the crude mixture afforded the phloroglucinol-based core 5 without further purification. Trifunctional core compound $^{54} \mathbf{6}$, was prepared (Scheme 4) by reacting 4-hydroxybenzaldehyde in the presence of sodium hydroxide with tris-(2-chloroethyl)-amine. The tris-aldehyde 14 was then treated with methylamine followed by reduction of the resulting tris-imine to afford the trifunctional core compound 6.

Synthesis of Trivalent Glycoclusters. Trisaccharide $7^{22 b}$ was coupled (Scheme 2) with trifunctional tris-methylamino cores $\mathbf{4 , 5}$, and $\mathbf{6}$ to prepare lactoside clusters by reductive amination in a phosphate-buffered saline solution ( $\mathrm{pH}$ 7.4) using $\mathrm{NaCNBH}_{3}$. Purification of the crude mixture by size-exclusion chromatography using a Sephadex resin allowed for the isolation and complete characterization of the lactoside clusters $\mathbf{8 , 9}$, and $\mathbf{1 0}$ by $1 \mathrm{D}$ and 2D NMR spectroscopies, as well as high resolution mass spectrometry.

Synthesis of Chitosan-Based Polymer. Trisaccharide $7^{22 \mathrm{~b}}$ was coupled with commerciallyavailable chitosan $\left(\mathrm{M}_{\mathrm{w}}=50,000\right)$ to yield the multivalent polymer 11 . The degree of substitution, determined by ${ }^{1} \mathrm{H}$ NMR spectroscopy, was 0.25 .

\section{Experimental Details}

General Methods. Tris-methylamino core compound $4,{ }^{52}$ trisaccharide $\mathbf{7},{ }^{22 b}$ and $\mathrm{CD} \mathbf{1}^{24}$ were prepared as described in the literature. All other chemicals were purchased from Aldrich and used as received. Solvents were used as purchased, except for $\mathrm{CH}_{2} \mathrm{Cl}_{2}$ (distilled from $\mathrm{CaH}_{2}$ ) and $\mathrm{MeOH}$ (distilled from Mg turnings). Analytical TLC was performed on silica gel $60-\mathrm{F}_{254}$ (Merck) with detection by fluorescence and/or by charring following immersion in a $5 \%$ $\mathrm{H}_{2} \mathrm{SO}_{4} / \mathrm{EtOH}$. Flash chromatography was performed with silica gel 60 (Silicycle). Preparative gel permeation chromatography was performed using (1) a $25 \square 900 \mathrm{~mm}$ column of Sephadex LH20 resin (Sigma), eluting with $\mathrm{MeOH}$ or (2) a $5 \square 100 \mathrm{~cm}$ column of Sephadex G-25 resin (Amersham Pharmacia Biotech), eluting with $5 \% \mathrm{nBuOH}$ in water. High resolution matrixassisted laser desorption ionization (HR-MALDI) mass spectra were recorded using dihydroxybenzoic acid as a matrix and external calibration using substance $\mathrm{P}$ on an IonSpec Ultima 7.4 Tesla FTMS instrument. ${ }^{1} \mathrm{H}$ and ${ }^{13} \mathrm{C}$ NMR spectra were recorded on a Brüker Avance 500 spectrometer (at $500 \mathrm{MHz}$ and $125 \mathrm{MHz}$, respectively) or Brüker Avance 600 spectrometer (at $600 \mathrm{MHz}$ and $150 \mathrm{MHz}$, respectively) at ambient temperature using $\mathrm{D}_{2} \mathrm{O}$ as the solvent.

1,3,5-Tris(2-hydroxyethoxy)benzene (12). Phloroglucinol (25.2 g, $0.2 \mathrm{~mol})$ was dissolved in DMF $(300 \mathrm{~mL})$ with ethylene carbonate $(70 \mathrm{~g}, 0.78 \mathrm{~mol})$ and $n \mathrm{Bu}_{4} \mathrm{NBr}(16 \mathrm{~g}, 76 \mathrm{mmol})$ to obtain a white suspension. The reaction mixture was heated at $150{ }^{\circ} \mathrm{C}$ for $16 \mathrm{~h}$. The dark brown mixture was cooled to ambient temperature then evaporated and the resulting syrup was purified by silica gel chromatography $\left(\mathrm{CH}_{2} \mathrm{Cl}_{2}\right.$ then $\mathrm{CH}_{2} \mathrm{Cl}_{2} / \mathrm{MeCN} 1: 1$ then $\left.\mathrm{MeCN}\right)$ to afford $12(18.1 \mathrm{~g}$, 
$35 \%$ ) as a yellow solid; ${ }^{1} \mathrm{H}$ NMR (400 MHz, $\left.\mathrm{CD}_{3} \mathrm{OD}\right): \square=3.84\left(\mathrm{t}, 6 \mathrm{H}, \mathrm{J}=4.8 \mathrm{~Hz}, \mathrm{CH}_{2} \mathrm{OH}\right), 3.99$ $\left(\mathrm{t}, 6 \mathrm{H}, J=4.8 \mathrm{~Hz}, \mathrm{OCH}_{2}\right), 6.16(\mathrm{~s}, 3 \mathrm{H}, P h) ;{ }^{13} \mathrm{C} \mathrm{NMR}\left(100 \mathrm{MHz}, \mathrm{CD}_{3} \mathrm{OD}\right): \square=60.3,69.2,93.9$, 160.8; HR-MALDI $m / z$ found 259.1175 $[M+\mathrm{H}]^{+}$. Calcd for $\mathrm{C}_{12} \mathrm{H}_{19} \mathrm{O}_{6} 259.1176$.

1,3,5-Tris(2-p-toluenesulfonyloxyethoxy)benzene (13). A solution of TsCl (16 g, 84 mmol) in $\mathrm{C}_{5} \mathrm{H}_{5} \mathrm{~N}(40 \mathrm{~mL})$ was added dropwise at $0{ }^{\circ} \mathrm{C}$ to a solution of $\mathbf{1 2}(4.5 \mathrm{~g}, 17.4 \mathrm{mmol})$ in $\mathrm{C}_{5} \mathrm{H}_{5} \mathrm{~N}(50 \mathrm{~mL})$. The reaction was stirred at ambient temperature for $16 \mathrm{~h}$ before pouring into $\mathrm{HCl}(1 \mathrm{M}, 250 \mathrm{~mL})$ and the aqueous layer was extracted with $\mathrm{CH}_{2} \mathrm{Cl}_{2}(3 \square 500 \mathrm{~mL})$. Organic layers were combined, dried $\left(\mathrm{Na}_{2} \mathrm{SO}_{4}\right)$ and concentrated under vacuum. The resulting solid was then washed with $\mathrm{CHCl}_{3} / \mathrm{EtOAc}(50: 1$, v/v) to afford $13(7.3 \mathrm{~g}, 58 \%)$ as a white solid. m.p. 135-137 ${ }^{\circ} \mathrm{C}$; ${ }^{1} \mathrm{H}$ NMR $\left(500 \mathrm{MHz},\left(\mathrm{CD}_{3}\right)_{2} \mathrm{CO}\right): \square=2.45$ (s, 9H, PhMe), 4.17 (t, 6H, J=4.3 Hz, $\left.\mathrm{OCH}_{2}\right), 4.38\left(\mathrm{t}, 6 \mathrm{H}, J=4.3 \mathrm{~Hz}, \mathrm{CH}_{2} \mathrm{OSO}_{2}\right), 5.94(\mathrm{~s}, 3 \mathrm{H}, P h), 7.48\left(\mathrm{~d}, 6 \mathrm{H}, J=8.2 \mathrm{~Hz}, P h \mathrm{SO}_{2}\right)$, $7.83(\mathrm{~d}, 6 \mathrm{H}, J=8.2 \mathrm{~Hz}, P h \mathrm{Me}) ;{ }^{13} \mathrm{C} \mathrm{NMR}\left(125 \mathrm{MHz},\left(\mathrm{CD}_{3}\right)_{2} \mathrm{CO}\right): \square=21.5,66.5,69.6,95.3$, 128.8, 130.9, 134.1, 146.0, 160.9; HR-FAB $m / z$ found $720.1397[M]^{+}, 721.1419[M+\mathrm{H}]^{+}$. Calcd for $\mathrm{C}_{33} \mathrm{H}_{36} \mathrm{O}_{12} \mathrm{~S}_{3} 720.1369, \mathrm{C}_{33} \mathrm{H}_{37} \mathrm{O}_{12} \mathrm{~S}_{3} 721.1447$, respectively.

1,3,5-Tris(2- $N$-methylaminoethoxy)benzene (5). A solution of $13(5.12 \mathrm{~g}, 7.1 \mathrm{mmol}), \mathrm{N}$ benzyl- $N$-methylamine $(18.3 \mathrm{~mL}, 142 \mathrm{mmol})$ and $\mathrm{K}_{2} \mathrm{CO}_{3}(1.96 \mathrm{~g}, 14.2 \mathrm{mmol})$ in dry THF (50 $\mathrm{mL}$ ) was heated at $85{ }^{\circ} \mathrm{C}$ for $16 \mathrm{~h}$ under an argon atmosphere. The reaction was poured into a saturated $\mathrm{NaHCO}_{3}$ solution $(250 \mathrm{~mL})$ and the aqueous layer was extracted with $\mathrm{CH}_{2} \mathrm{Cl}_{2}(3 \square 250$ $\mathrm{mL})$. Organic layers were combined, dried $\left(\mathrm{Na}_{2} \mathrm{SO}_{4}\right)$ and concentrated under vacuum. The crude product was dissolved in $\mathrm{THF} / \mathrm{H}_{2} \mathrm{O}(1: 1, \mathrm{v} / \mathrm{v})$ and $\mathrm{Pd}-\mathrm{C} 10 \%(2.5 \mathrm{~g})$ was added. The reaction was vigorously stirred under a hydrogen atmosphere for $16 \mathrm{~h}$. The suspension was then filtered through a celite pad and the filtrate evaporated to afford $5(1.48 \mathrm{~g}, 70 \%)$ as a yellow gum. ${ }^{1} \mathrm{H}$ NMR (500 MHz, CD 3 OD): $\square=1.54$ (bs, 3H, NHMe), 2.37 (s, 9H, NHMe), 2.82 (t, J = 5.1 Hz, $\left.6 \mathrm{H}, \mathrm{CH}_{2} \mathrm{~N}\right), 3.90\left(\mathrm{t}, 6 \mathrm{H}, J=5.1 \mathrm{~Hz}, \mathrm{OCH}_{2}\right), 6.00(\mathrm{~s}, 3 \mathrm{H}, P h) ;{ }^{13} \mathrm{C} \mathrm{NMR}\left(125 \mathrm{MHz}, \mathrm{CD}_{3} \mathrm{OD}\right): \square=$ 36.2, 50.6, 67.0, 94.0, 160.5; HR-MALDI $\mathrm{m} / z$ calcd for $\mathrm{C}_{15} \mathrm{H}_{28} \mathrm{~N}_{3} \mathrm{O}_{3}$ 298.2125, found 298.2123 $[M+\mathrm{H}]^{+}$.

Tris[2-(4-formylphenoxy)ethyl]amine (14). Sodium hydroxide (1.33 g, $33.2 \mathrm{mmol})$ was added to a solution of 4-hydroxybenzaldehyde (3.04 g, $24.9 \mathrm{mmol})$ in EtOH $(200 \mathrm{~mL})$. The mixture was stirred at ambient temperature for $1 \mathrm{~h}$ then tris-(2-chloroethyl)amine hydrochloride ( $2 \mathrm{~g}, 8.3 \mathrm{mmol}$ ) was added and the reaction was heated to reflux $\left(90^{\circ} \mathrm{C}\right)$ for $3 \mathrm{~h}$. The solution was then cooled to ambient temperature, poured into water $(600 \mathrm{~mL})$ and filtered to afford 14 (3.29 g, 86\%) as a white powder. m.p. $80-82{ }^{\circ} \mathrm{C} ;{ }^{1} \mathrm{H}$ NMR $\left(400 \mathrm{MHz}, \mathrm{CDCl}_{3}\right)$ : $\square=3.20(\mathrm{t}, 6 \mathrm{H}, J$ $\left.=5.6 \mathrm{~Hz}, \mathrm{NCH}_{2}\right), 4.17\left(\mathrm{t}, 6 \mathrm{H}, J=5.6 \mathrm{~Hz}, \mathrm{CH}_{2} \mathrm{O}\right), 6.95(\mathrm{~d}, 6 \mathrm{H}, J=8.7 \mathrm{~Hz}, P h \mathrm{O}), 7.95(\mathrm{~d}, 6 \mathrm{H}, J=$ 8.7 Hz, PhCHO), 9.86 (s, 3H, CHO); ${ }^{13} \mathrm{C} \mathrm{NMR}\left(\mathrm{CDCl}_{3}, 100 \mathrm{MHz}\right): \square=54.3,67.3,114.7,130.1$, 131.9, 163.6, 190.7; HR-MALDI $\mathrm{m} / z$ found $484.1711[M+\mathrm{Na}]^{+}$, found $462.1893[M+\mathrm{H}]^{+}$. Calcd for $\mathrm{C}_{27} \mathrm{H}_{27} \mathrm{NNaO}_{6} 484.1731, \mathrm{C}_{27} \mathrm{H}_{28} \mathrm{NO}_{6} 462.1911$, respectively.

Tris\{2-[4-( $N$-methylamino)methyl-phenoxy]ethyl\}amine (6). A solution of 14 (250 mg, $0.54 \mathrm{mmol})$ in methylamine $(2 \mathrm{M}$ in THF, $13.5 \mathrm{~mL}, 27 \mathrm{mmol})$ was stirred at ambient temperature for $4 \mathrm{~h}$ then poured into water $(100 \mathrm{~mL})$. The aqueous layer was extracted with $\mathrm{CH}_{2} \mathrm{Cl}_{2}(3 \square 150$ $\mathrm{mL})$, organic layers were dried $\left(\mathrm{Na}_{2} \mathrm{SO}_{4}\right)$, filtered and evaporated under vacuum. The crude mixture was dissolved in $\mathrm{MeOH}(50 \mathrm{~mL})$ and $\mathrm{NaBH}_{4}(600 \mathrm{mg}, 15.78 \mathrm{mmol})$ was added. The reaction was stirred at ambient temperature for $16 \mathrm{~h}$ before pouring into water $(100 \mathrm{~mL})$. The 
aqueous layer was extracted with $\mathrm{CH}_{2} \mathrm{Cl}_{2}(3 \square 150 \mathrm{~mL})$, organic layers were dried $\left(\mathrm{Na}_{2} \mathrm{SO}_{4}\right)$, filtered and evaporated under vacuum to afford $6(210 \mathrm{mg}, 76 \%)$. ${ }^{1} \mathrm{H}$ NMR $\left(500 \mathrm{MHz}, \mathrm{CDCl}_{3}\right)$ : $\mathrm{C}=2.42$ (s, 9H, NMe), 3.14 (brt, $6 \mathrm{H}, \mathrm{NCH}_{2} \mathrm{CH}_{2} \mathrm{O}$ ), 3.66 (s, 6H, $\mathrm{CH}_{2} \mathrm{NHMe}$ ), 4.08 (brt, $6 \mathrm{H}$, $\mathrm{NCH}_{2} \mathrm{CH}_{2} \mathrm{O}$ ), 6.83 (brd, 6H, PhO), 7.19 (brd, 6H, PhCHO); Selected ${ }^{13} \mathrm{C}$ NMR (125 MHz, $\left.\mathrm{CDCl}_{3}\right): \mathrm{C}=35.9,54.4,55.4,66.8,114.8,129.3,132.3,158.1$; HR-MALDI $\mathrm{m} / \mathrm{z}$ found 529.3119 $[M+\mathrm{Na}]^{+}$. Calcd for $\mathrm{C}_{30} \mathrm{H}_{42} \mathrm{~N}_{4} \mathrm{NaO}_{3} 529.3149$.

Lactose Cluster 8. A solution of core compound $\mathbf{4}^{52}(114 \mathrm{mg}, 0.61 \mathrm{mmol})$, lactose trisaccharide $7^{22 \mathrm{~b}}(1.222 \mathrm{~g}, 2.42 \mathrm{mmol})$ and $\mathrm{NaCNBH}_{3}(343 \mathrm{mg}, 5.46 \mathrm{mmol})$ in phosphate buffered saline $(10 \mathrm{~mL})$ was stirred at ambient temperature for 7 days after adjusting the $\mathrm{pH}$ to 7 with $\mathrm{AcOH}$. The reaction mixture was freeze dried from $\mathrm{H}_{2} \mathrm{O}$ and the crude mixture was purified using gel permeation chromatography (Sephadex G-25: $5 \% n \mathrm{BuOH}$ in $\mathrm{H}_{2} \mathrm{O}$ ) affording 8 (810 $\mathrm{mg}, 82 \%)$ as an amorphous solid. Selected ${ }^{1} \mathrm{H}$ NMR (600 MHz, $\left.\mathrm{D}_{2} \mathrm{O}\right)$ : $\mathrm{\square}=2.45-2.60$ (brs, $9 \mathrm{H}$, $\mathrm{N} M e), 4.35\left(\mathrm{~d}, 3 \mathrm{H}, J_{1,2}=7.8 \mathrm{~Hz}\right), 4.45\left(\mathrm{~d}, 3 \mathrm{H}, J_{1,2}=7.8 \mathrm{~Hz}\right)$; HR-MALDI $m / z$ found 1675.7120 $[M+\mathrm{Na}]^{+}$. Calcd for $\mathrm{C}_{63} \mathrm{H}_{120} \mathrm{~N}_{4} \mathrm{NaO}_{45} 1675.7122$.

Lactose Cluster 9. A solution of the phloroglucinol-based core 5 (192 mg, $0.64 \mathrm{mmol}$ ), lactose trisaccharide $7^{22 \mathrm{~b}}(1.3 \mathrm{~g}, 2.58 \mathrm{mmol})$ and $\mathrm{NaCNBH}_{3}(366 \mathrm{mg}, 5.82 \mathrm{mmol})$ in phosphate buffered saline $(10 \mathrm{~mL})$ was stirred at ambient temperature for $7 \mathrm{~d}$ after adjusting the $\mathrm{pH}$ to 7 with $\mathrm{AcOH}$. The reaction mixture was freeze dried from $\mathrm{H}_{2} \mathrm{O}$ and the crude mixture was purified using gel permeation chromatography (Sephadex G-25: 5\% nBuOH in $\mathrm{H}_{2} \mathrm{O}$ ) affording 9 (1.06 g, 93\%) as an amorphous solid. Selected ${ }^{1} \mathrm{H}$ NMR $\left(\mathrm{D}_{2} \mathrm{O}, 600 \mathrm{MHz}\right)$ : $\mathrm{\square}=2.78$ (brs, $\left.9 \mathrm{H}, \mathrm{NMe}\right), 4.34$ $(\mathrm{d}, 3 \mathrm{H}, J=7.8 \mathrm{~Hz}), 4.43$ (d, 3H, $J=7.9 \mathrm{~Hz}), 6.26$ (brs, 3H, Ph); Selected ${ }^{13} \mathrm{C}$ NMR $\left(\mathrm{D}_{2} \mathrm{O}, 150\right.$ MHz): $\square=41.6,102.6,103.0$; HR-MALDI $\mathrm{m} / z$ found $1784.7185[M+\mathrm{Na}]^{+}$. Calcd for $\mathrm{C}_{69} \mathrm{H}_{123} \mathrm{~N}_{3} \mathrm{NaO}_{48} 1784.7174$.

Lactose Cluster 10. A solution of core compound 6 (200 $\mathrm{mg}, 0.39 \mathrm{mmol})$, lactose trisaccharide $7^{22 \mathrm{~b}}(995 \mathrm{mg}, 1.97 \mathrm{mmol})$ and $\mathrm{NaCNBH}_{3}(223 \mathrm{mg}, 3.55 \mathrm{mmol})$ in phosphate buffered saline $(10 \mathrm{~mL})$ was stirred at ambient temperature for 7 days after adjusting the $\mathrm{pH}$ to 7 with $\mathrm{AcOH}$. The reaction mixture was freeze dried from $\mathrm{H}_{2} \mathrm{O}$ and the crude mixture was purified using gel permeation chromatography (Sephadex G-25: 5\% $n \mathrm{BuOH}$ in $\mathrm{H}_{2} \mathrm{O}$ ) affording 10 (700 $\mathrm{mg}, 88.9 \%$ ) as an amorphous solid. Selected ${ }^{1} \mathrm{H}$ NMR (500 MHz, $\left.\mathrm{D}_{2} \mathrm{O}\right): \mathrm{\square}=2.69$ (s, 9H, NMe), $4.34(\mathrm{~d}, 3 \mathrm{H}, J=7.8 \mathrm{~Hz}), 4.43(\mathrm{~d}, 3 \mathrm{H}, J=7.9 \mathrm{~Hz}), 6.88(\mathrm{~m}, 6 \mathrm{H}), 7.23-7.33(\mathrm{~m}, 6 \mathrm{H})$; selected ${ }^{13} \mathrm{C}$ NMR (125 MHz, $\mathrm{D}_{2} \mathrm{O}$ ): $\mathrm{\square}=102.4,102.8,115.1,131.4,132.4,132.8,159.0$; HR-MALDI $\mathrm{m} / \mathrm{z}$ found $1993.8377[M+\mathrm{Na}]^{+}$. Calcd for $\mathrm{C}_{84} \mathrm{H}_{138} \mathrm{~N}_{4} \mathrm{NaO}_{48} 1993.8373$.

Synthesis of Chitosan-Based Polymer 11. ${ }^{40 \mathrm{c}}$ Chitosan $(100 \mathrm{mg})$ was added to $\mathrm{pH} 7.4$ phosphate buffered saline solution $(50 \mathrm{~mL})$ and acetic acid (5-8 drops) was added until the chitosan dissolved. Trisaccharide 7 (166 mg, 50 mmol, 0.8 eq.) and $\mathrm{NaCNBH}_{3}(100 \mathrm{mg}, 1.5$ $\mathrm{mmol}, 2.4$ eq.) were then added and the solution was stirred for $7 \mathrm{~d} . \mathrm{K}_{2} \mathrm{CO}_{3}(250 \mathrm{mg})$ was added, producing a precipitate, which was alternately centrifuged and washed 3 times with water. It was then dried under vacuum, yielding a white solid $(311 \mathrm{mg})$. A portion of this was redissolved in $1 \mathrm{M} \mathrm{HCl}$, and was then evaporated in vacuo to give a new white solid. The degree of substitution was determined to be 0.25 from the ratio of the ${ }^{1} \mathrm{H}$ NMR peak at $4.42(\mathrm{H}-1$ or H-1' of lactose) and 4.8-5.1 (H-1 of GlcN). Selected ${ }^{1} \mathrm{H}$ NMR (500 MHz, $\left.\mathrm{D}_{2} \mathrm{O}\right)$ 3.3-3.4 (br m, 1H, H- 
$2 \mathrm{GlcN}$ ), 3.5-3.6 (br m, 1.6H, H-2 of $N$-alkyl GlcN), 4.43 (d, $0.5 \mathrm{H}, \mathrm{H}-1$ or H-1' lactose), 4.54 (d, 0.7H, H-1 or H-1' lactose), 4.8-4.9 (br, 1H, H-1 GlcN), 5.0-5.1 (br, 0.7H, H-1 (N-alkyl)GlcN).

Preparation of Polyviologen 2. ${ }^{\text {la }}$ 1,10-Dibromodecane $(2.6 \mathrm{~g}, 16.7 \mathrm{mmol})$ and 4,4' bipyridine (5 g, $16.7 \mathrm{mmol})$ were dissolved in 1:1 MeOH/DMF $(16 \mathrm{~mL})$, and stirred for $20 \mathrm{~h}$ at $75{ }^{\circ} \mathrm{C}$. The reaction mixture was then diluted in $\mathrm{H}_{2} \mathrm{O}(50 \mathrm{~mL})$, then extracted with $\mathrm{CHCl}_{3}(50$ $\mathrm{mL})$. The aqueous layer was then concentrated in vacuo to afford a dark yellow solid $(6.5 \mathrm{~g}) .{ }^{1} \mathrm{H}$ NMR (500 MHz, $\left.\mathrm{D}_{2} \mathrm{O}\right) \square=1.13-1.5\left(\mathrm{~m}, \mathrm{H}_{\mathrm{E}-\mathrm{G}}\right), 2.12$ (br s, $\mathrm{H}_{\mathrm{D}}$ ), 4.68 (br t, $\mathrm{H}_{\mathrm{C}}, J=7.4 \mathrm{~Hz}$ ), 7.98 (br d, $\mathrm{H}_{\mathrm{B}}$ from endgroups), 8.34 (br d, $\mathrm{H}_{\mathrm{B}^{\prime}}$ from endgroups), $8.51\left(\mathrm{~d}, \mathrm{H}_{\mathrm{B}}, J=6.4 \mathrm{~Hz}\right.$ ), 8.78 (br s, $\mathrm{H}_{\mathrm{A}}$ from endgroups), 8.94 (d, $\mathrm{H}_{\mathrm{A}^{\prime}}$ from endgroups), $9.08\left(\mathrm{~d}, \mathrm{H}_{\mathrm{A}}, J=6.4 \mathrm{~Hz}\right)$.

Preparation of Pseudopolyrotaxane 3. The polyviologen $2(4.5 \mathrm{mg}, 0.58 \square \mathrm{mol})$ was added to $0.5 \mathrm{~mL}$ of a $20 \mathrm{mM}$ solution of CD 1. ${ }^{24}$ This solution was allowed to stand $4 \mathrm{~d}$ at ambient temperature. The threading process was monitored by TLC using 4:3:3:2 EtOAc/MeOH/H${ }_{2} \mathrm{O} / \mathrm{AcOH}$ and $5 \% \mathrm{H}_{2} \mathrm{SO}_{4}$ in EtOH as the developing agent. This stock solution, $(20 \mathrm{mM}$ per molar lactose) was diluted accordingly to provide the appropriate per molar lactose concentrations for the agglutination assay.

Agglutination Assay. Lactoside (6.26 $\square$ final concentration) and recombinant human galectin- $1^{51}(62.6 \square \mathrm{M})$ were mixed in phosphate-buffered saline $(8 \square \mathrm{L})$ at room temperature for 10 minutes. $42 \square \mathrm{L}$ of a solution of $7.5 \times 10^{6} \mathrm{CEM}$ cells $/ \mathrm{mL}$ in serum-free RPMI 1640 media were added. The mixture $\left(10 \square \mathrm{M}\right.$ galectin- $1,3.15 \times 10^{5}$ cells) was allowed to stand for $5 \mathrm{~min}$ at $37^{\circ} \mathrm{C}$. The mixture was vortexed briefly and a $10 \square \mathrm{L}$ aliquot taken for imaging by light microscopy. At least three independent experiments were performed for each lactoside concentration and for the controls: no galectin-1, no lactoside, polymer $\mathbf{2}$, and the pseudopolyrotoxane of $\mathbf{2}$ with native $\mathrm{C}$-cyclodextrin. CEM cells (subclone of ATCC \#CCL119) were grown in RPMI 1640 medium, supplemented with $10 \%$ fetal bovine serum, $10 \mathrm{mM}$ HEPES, and $2 \mathrm{mM} \mathrm{L-glutamine} \mathrm{at} 37^{\circ} \mathrm{C}$ and $5 \% \mathrm{CO}_{2}$.

\section{References}

(22) (a) Gestwicki, J. E.; Cairo, C. W.; Strong, L. E.; Oetjen, K. A.; Kiessling, L. L. J. Am. Chem. Soc. 2002, 124, 14922-14933. (b) Kalovidouris, S. A.; Blixt, O.; Nelson, A.; Vidal, S.; Turnbull, W. B.; Paulson, J. C.; Stoddart, J. F. J. Org. Chem. 2003, 68, 8485-8493. (c) Kitov, P. I.; Shimizu, H.; Homans, S. W.; Bundle, D. R. J. Am. Chem. Soc. 2003, 125, 3284-3294.

(24) Nelson, A.; Stoddart, J. F. Org. Lett. 2003, 5, 3783-3786.

(40) (a) Sashiwa, H.; Makimura, Y.; Shigemasa, Y.; Roy, R. Chem. Commun. 2000, 909-910. (b) Sashiwa, H.; Thompson, J.; Das, S. K.; Shigemasa, Y.; Tripathy, S.; Roy, R. Biomacromolecules 2001, 1, 303-305. (c) Sashiwa, H.; Shigemasa, Y.; Roy, R. Bull. Chem. Soc. Jpn. 2001, 74, 937-943.

(41) (a) Harada, A.; Adachi, H.; Kawaguchi, Y.; Okada, M.; Kamachi, M. Polym. J. (Tokyo) 1996, 28, 159-163. (b) Herrmann, W.; Keller, B.; Wenz, G. Macromolecules 1997, 30, 4966-4972.

(51) Pace, K. E.; Hahn, H. P.; Baum, L. G. Methods Enzymol. 2003, 363, 499-518. 
(52) Turnbull, W. B.; Kalovidouris, S. A.; Stoddart, J. F. Chem. Eur. J. 2002, 8, 2988-3000.

(53) Bradshaw, J. S.; Krakowiak, K. E. J. Heterocyclic Chem. 1998, 35, 519-524.

(54) Kaliappa, G. R.; Shukla, R.; Mishra, S.; Bharadwaj, P. K. Tetrahedron Lett. 1993, 34, 5631-5634. 